\title{
Calibration and Performance of the Precision Chambers of the ATLAS Muon Spectrometer
}

\author{
M. Iodice, INFN Roma Tre \\ on behalf of the ATLAS Collaboration
}

\begin{abstract}
The ATLAS Muon Spectrometer consists of a system of precision tracking and trigger chambers embedded in a 2-8 Tm magnetic field generated by three large air-core superconducting toroids. The precision Monitored Drift Tube (MDT) chambers measure the track sagitta up to a pseudorapidity of 2.7 with a $40 \mu \mathrm{m}$ uncertainty yielding a design muon transverse momentum resolution of $10 \%$ at $1 \mathrm{TeV}$. Muon tracking is complemented in the very forward region by Cathode Strip Chambers (CSC). The calibration program, essential to achieve the spectrometer design performance and physics reach, is conducted at three worldwide computing centers. These centers each receive a High Level Trigger data stream that enables high statistics determination of timing offset constants and drift-time to drift-space conversion relationship. During the LHC data taking periods in $\mathbf{2 0 1 0}$ and 2011 , a system of periodic calibration updates has been established. The calibration algorithms, methods and tools and performance results for this first period of LHC collision data will be presented.
\end{abstract}

\section{INTRODUCTION}

$66 \bigcirc$ ENEVA, 31 October 2011. After some 180 days of running and four hundred trillion proton proton collisions, the LHCs 2011 proton run came to an end at 5.15 pm yesterday evening. For the second year running, the LHC team has largely surpassed its operational objectives, steadily increasing the rate at which the LHC has delivered data to the experiments".

This is the first sentence of the press release sent by the Director of CERN just after the closure of the IEEE NSS 2011 Conference, clearly stating the great success of a first period of operation of a machine and detectors of unprecedented complexity and performance.

ATLAS is a multipurpose detector [1], [2] installed at the Large Hadron Collider (LHC) at CERN where two proton beams collide at a center-of-mass-energy of $7 \mathrm{TeV}$. An integrated luminosity of almost $6 \mathrm{fb}^{-1}$ have been delivered so far to the experiment. In this paper the focus is on the procedures of calibrations of the precision chambers of the ATLAS Muon Spectrometer established and carried out during these first years of data taking. The calibration methods, tools and performance will be presented.

\section{The AtLAS Muon Spectrometer: Detector COMPONENTS AND EXPECTED PERFORMANCE}

The ATLAS Muon Spectrometer (MS) is the external part of the detector and defines its large volume. In the central part (barrel), it extends from an internal radius of $4.5 \mathrm{~m}$ to the external radius of $11 \mathrm{~m}$. The distance along the beam line of the two forward (end-cap) detectors is $40 \mathrm{~m}$. The magnetic field is generated by a system of three superconducting toroids in air, one for barrel and one for each of two end-caps. The muon system is divided in four regions: two barrel sides (along the beam axis and divided at the vertex of interaction, also referred to as Barrel side $\mathrm{A}$ and Barrel side $\mathrm{C}$ regions) and the two end-cap regions (Endcap-A and Endcap-C).

Tracking and precision chambers are arranged in three stations (inner, middle, outer). For most of the acceptance the precision tracking chambers consist of Monitored Drift Tubes (MDT). In the most forward region of the end-cap inner regions, the Cathode Strip Chambers (CSC) are used since they are able to cope with higher background rates. The trigger chambers are based on two technologies: the Resistive Plate Chambers (RPC) instrument the barrel while the Thin Gap Chambers (TGC) are used in the higher background environment of the end-caps.

The momentum of muons is determined by measuring the curvature of the track with a field integral varying between 2 and $8 \mathrm{Tm}$. The muon track curvature is measured with the three precision chamber stations positioned along its trajectory.

In Fig. 1 the expected performance in terms of the muon transverse momentum resolution is shown, together with the individual contributions [3]. A design overall resolution of less than $5 \%$ for $\mathrm{p}_{T}<300 \mathrm{GeV}$ and $\sim 10 \%$ at $1 \mathrm{TeV}$, can be achieved. At large momenta $\left(\mathrm{p}_{T}>300 \mathrm{GeV}\right)$ the main contribution comes from the intrinsic resolution of the precision chambers, their calibrations and alignment.

To achieve the design momentum resolution, precision chambers should measure the muon position in the bending plane with an accuracy better than $40 \mu \mathrm{m}$. For the MDT this leads to a single point (tube) average spatial resolution of about $100 \mu \mathrm{m}$ (6/8 measurements points per chamber). Also, calibrations and alignment should have a precision better than $40 \mu \mathrm{m}$.

\section{The Monitored Drift Tubes Chambers And PRINCIPLES OF CALIBRATION}

The Monitored Drift Tube (MDT) chambers [4] are made of staggered layers of high-pressure drift tubes. The aluminum tubes have a diameter of $3 \mathrm{~cm}$ with a $50 \mu \mathrm{m}$ diameter goldplated W-Re anode wire at the center. Chambers are operated with a $\mathrm{Ar}: \mathrm{CO}_{2}$ (93:7) gas mixture at 3 bar absolute pressure, in avalanche mode with a relatively low gas gain to avoid aging effects. At the operating voltage of $3080 \mathrm{~V}$ the gas gain is $\sim 2 \times 10^{4}$ and the maximum drift time is $\sim 700 \mathrm{~ns}$. 


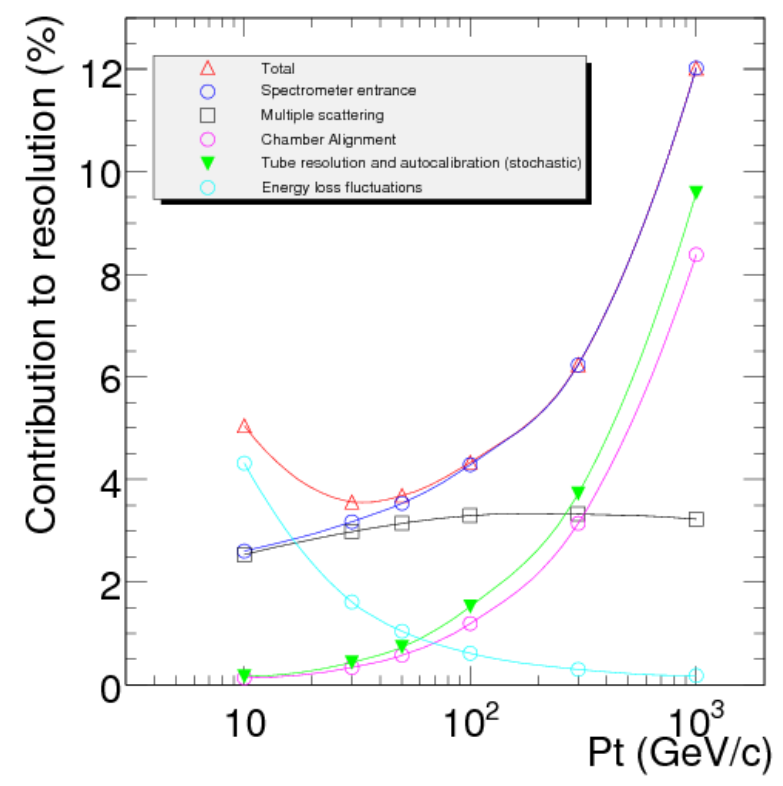

Fig. 1. Contributions to the muon transverse momentum resolution of the ATLAS Muon Spectrometer [3].

The chambers have an internal alignment system to monitor deformations as well as sensors monitoring position relative to other chambers.

Fig. 2 shows a photograph of one Barrel Inner MDT chamber, before service mounting (electronics, gas distribution system) now installed in the spectrometer. It consists of two multilayers, each composed of four staggered layers of 36 tubes $270 \mathrm{~cm}$ long.

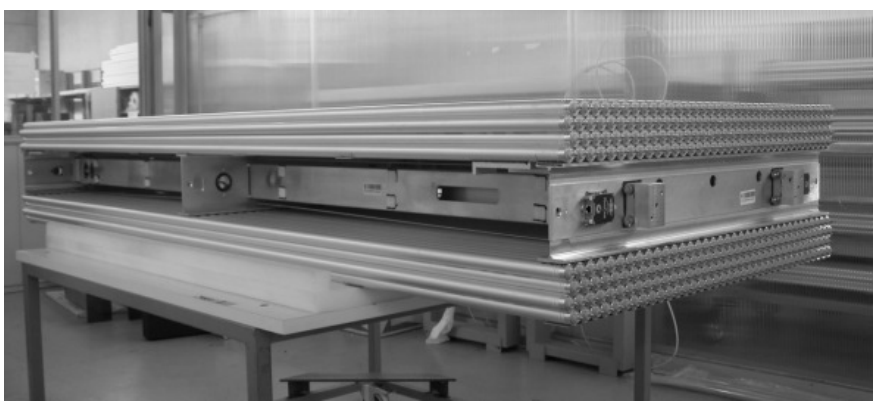

Fig. 2. Photograph of a MDT chamber made of two multilayers each of four layers of staggered drift tubes.

When a charged particle traverses a MDT chamber, the crossed tubes provide a direct measurement of the arrival time of drift electrons to the wire with respect to the trigger of the event. Thus, the basic principles of the calibrations are the following:

- determination of individual tube offsets, also referred to as $t_{0}$ 's per channel. Several sources contribute to these time offsets: signal detection threshold settings, electronic delays (cables, electronic boards, other sources from data acquisition), time of flight of particles from the interaction point (IP), propagation of the signal along the tube;

- Determination of electron avalanche drift-time (the arrival time of drift electrons with respect to the time the particle traverse the tube);

- Conversion of the drift-time to drift-distance accounting for temperature and Lorentz angle corrections to the electron drift paths (the latter being of relevance since there are regions with strong magnetic field gradients also within one chamber volume). This is accomplished through the drift-time to radius relationship, also referred to as the $r(t)$ relation.

- Determination of the tube spatial resolution as a function of the distance from the wire to provide proper hit weights to the tracking algorithms.

Once tube hits are calibrated, track-segments on a single chamber can be reconstructed with the best fit of a straight (or curved) segment tangent to all drift radii of tubes in the track pattern.

Thus, the main ingredients of the calibrations are the determination of individual channel timing offset $\left(t_{0}\right.$ 's) and the $r(t)$ relations for all chambers [5]. Essential input to the calibrations is also the knowledge of the current temperature and magnetic field maps. These are continuously measured through sensors positioned on the chambers.

In Fig. 3 the drift time spectrum of tubes in one chamber is shown. The minimum drift time $t_{0}$ (corresponding to muons passing close to the tube wire) and the maximum drift time $t_{\max }$ (corresponding to muons passing close to the tube wall) are determined by a fit to the leading and trailing edge of the time distribution, respectively.

In Fig. 4 the $r(t)$ relation of one chamber is shown, as determined via an iterative procedure of track residuals minimization, also referred to as the "autocalibration" procedure.

The autocalibration works as follows. At each step of the procedure the $r(t)$ relation determined in the previous iteration (or a starting trial $r(t)$ relation for the first pass) is used to convert the drift time measurements into drift radii for use in a straight line fit. The average difference between the drift radius obtained using this $r(t)$ relation and the distance of closest approach to the wire of the fitted track (the residual), would be zero at all radii if the $r(t)$ relation were exact. Deviations from zero indicate a mis-calibrations and the average deviation itself, at a given drift-time, can be directly used as a correction to the $r(t)$. After a few iterations the $r(t)$ relation converges to a stable solution. Additional corrections are applied to compensate for temperature and magnetic field effects.

It must be considered that both the drift time spectrum and the $r(t)$ relations depend on ambient and operational parameters (temperature, magnetic field, gas composition), hence frequent monitoring of the stability of the calibration constants is needed. The objective in ATLAS is to perform daily calibrations with a sample of about 30M muon tracks.

\section{A. The Muon Calibration Stream}

The data flow in ATLAS is structured in three levels of trigger, designed to record events at approximately $200 \mathrm{~Hz}$ from the LHCs $40 \mathrm{MHz}$ bunch crossing rate. The first level 


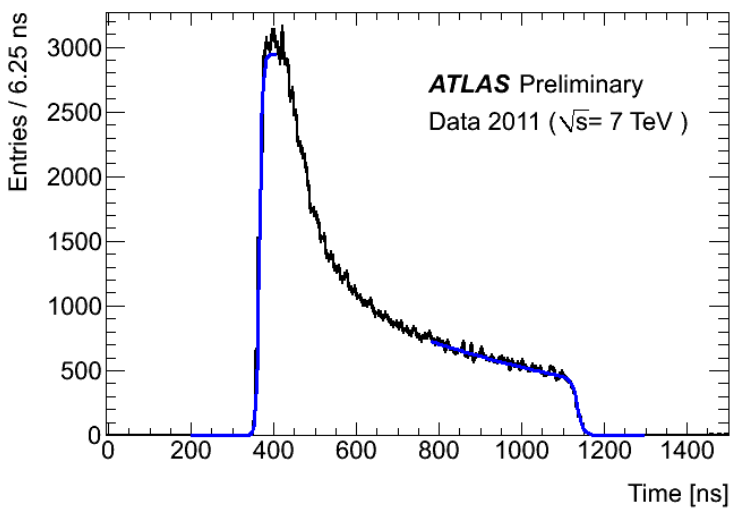

Fig. 3. Drift time distribution of tubes in one MDT chamber [9].

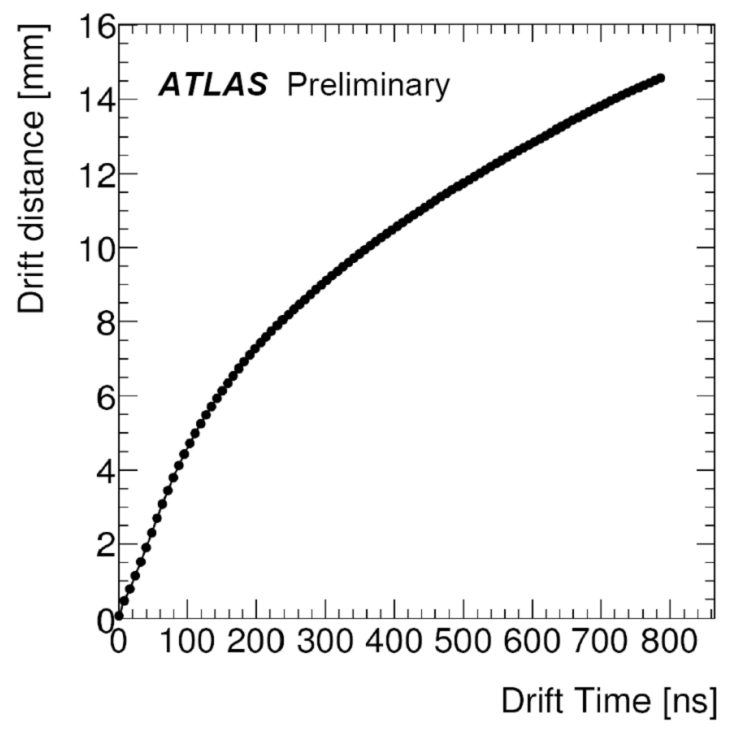

Fig. 4. $r(t)$ relation in one MDT chamber [9].

trigger is a hardware-based system using information from the calorimeter and muon sub-detectors. It reduces the input rate to a maximum of about $75 \mathrm{kHz}$. The second (L2) and third (Event Filter, EF) levels are software-based systems using information from all sub-detectors. Together, L2 and EF are called the High Level Trigger. After the event filter a maximum rate of muon triggered events of about $40 \mathrm{~Hz}$ is recorded on tape.

Standard data flow is thus not sufficient for muon calibration purposes, aiming at collecting enough statistics to allow a calibration per day with a sample of $\sim 30 \times 10^{6}$ muon tracks. Accounting for data taking efficiency the requirement is an acquisition rate of about $1 \mathrm{kHz}$.

A dedicated muon calibration stream is therefore enabled to extract muon selected events at the second level trigger with the required statistics, at about $4 \mathrm{kHz}$ [6]. Such a stream is sent to three calibration centers, in Rome, Munich, Michigan (three Tier2 grid farms in the ATLAS computing system), where data are processed with enough computing power $(\sim 100 \mathrm{CPUs})$ to provide calibration constants in time for reconstruction (36 hours). In Fig. 5 a scheme of the extraction of the muon calibration stream is shown.

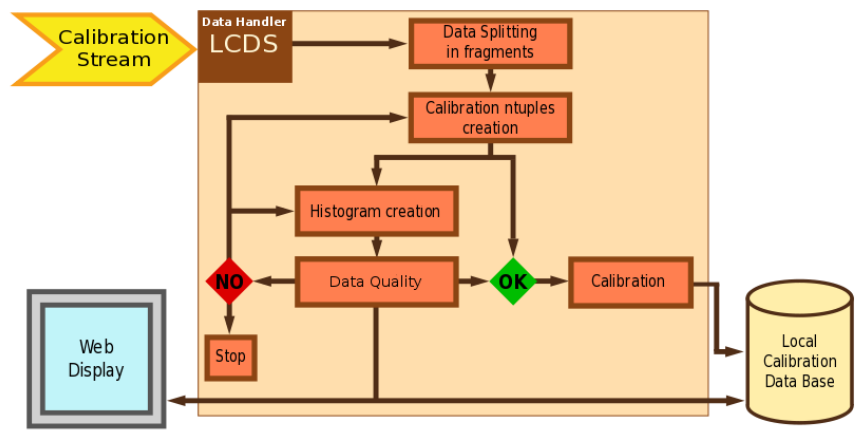

Fig. 5. Calibration stream processing at ATLAS calibration centers.

Such a dedicated muon stream also represents a privileged source of high statistics muon data to allow a detailed analysis of MDT chambers data quality assessment. At the calibration centers, shifters continuously control and monitor the data quality and calibration processing via web-interface.

\section{B. Precision and Stability of the Calibration constants}

The statistical uncertainty on the determination of the $t_{0}$ from the fit of the raising edge of the drift time spectrum, as a function of the number of entries in the spectrum is shown in Fig. 6. The statistical uncertainty goes well below $1 \mathrm{~ns}$ for a number of entries larger than few thousand. The requirement is to have at least $10 \mathrm{k}$ hits for the fits yielding a typical error of $0.5 \mathrm{~ns}$, corresponding to a spatial uncertainty of about 10 $\mu \mathrm{m}$. The calibration procedure is such that if a single tube tube does not reach the required statistics, tubes are grouped in increasing numbers, following the front-end read-out architecture: single tube, grouping per "mezzanine" (24 tubes in the same front-end electronic card), per multilayer $(\mathcal{O}(100)$ tubes), per chamber (several hundreds tubes, depending on the chamber).

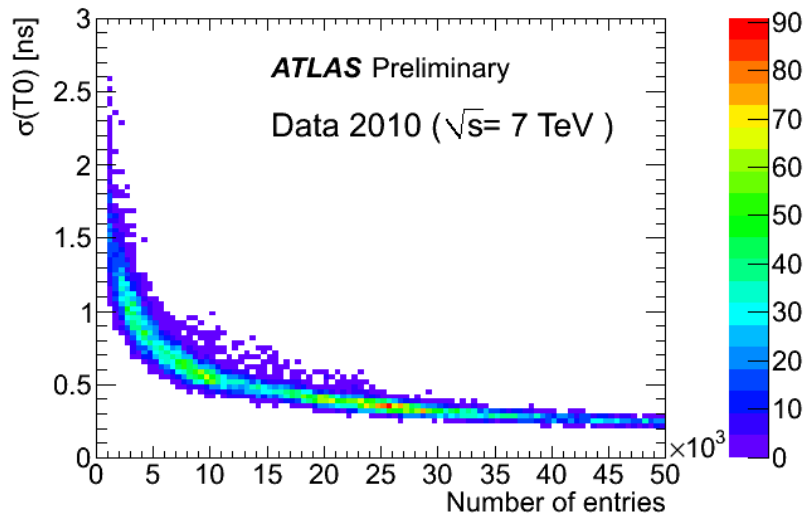

Fig. 6. The statistical error on the $t_{0}$ fits as a function of number hits in the time spectrum [9].

The reproducibility and stability of the calibration constants were tested, comparing results obtained in different high statistics runs. In Fig. 7 the change in $t_{0}$ over a 2 months 
period in 2010 for 1118 MDT chambers is shown. Each entry represents the weighted average $t_{0}$ for one chamber. The width of the distribution is below $1 \mathrm{~ns}$, compatible with the statistical uncertainty of each measurement. The measured mean variation of $-0.3 \mathrm{~ns}$ is compatible with drifts of the clock phase due to environmental conditions (monitored and compensated, when necessary, by the ATLAS trigger group, with adjustments of fraction of a nanosecond).

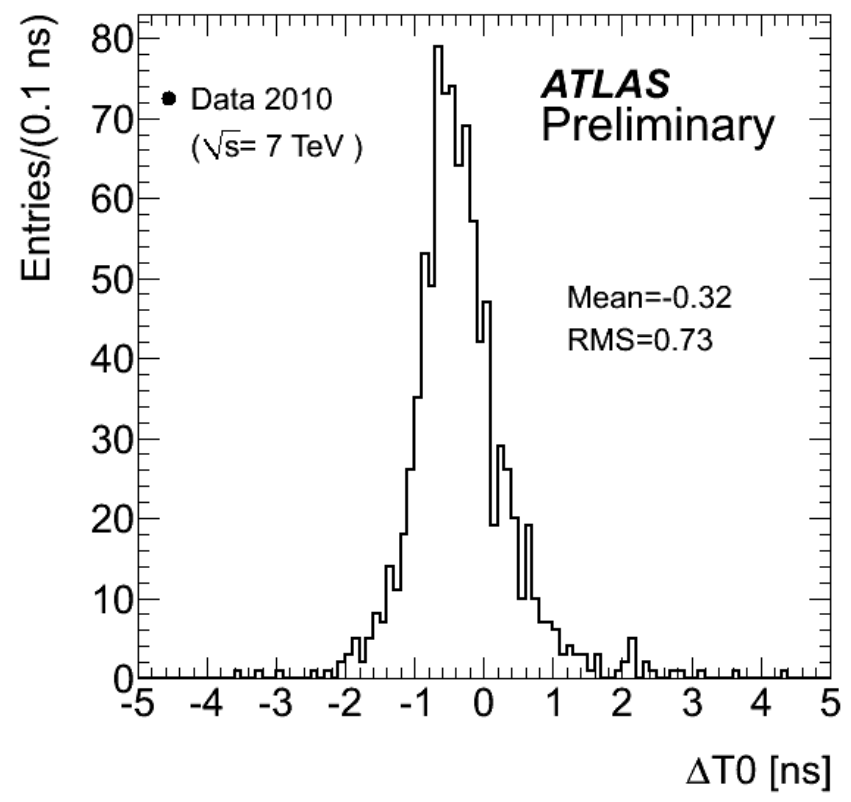

Fig. 7. The difference between t0s for all MDT chambers over a 2-month period in 2010 [9].

The comparison between the $r(t)$ relations measured in two different high statistics runs taken in October 2010, few days apart, is shown in Fig. 8. The $r(t)$ relations are evaluated per chamber for each run. In each time bin, the distribution of the differences between the two $r(t)$ 's is fitted with a gauss function. In the two plots, respectively for barrel (top) and endcap (bottom), the means of the gaussians are shown (black dots). The colored bands represent the spread of the $r(t)$ differences around the mean value, i.e. the $\pm 1 \sigma$ regions where $\sigma$ is the width of the gaussian distributions in that bin. The plots show the short-term reproducibility and stability of the $r(t)$ determination that is within $\pm 10 \mu \mathrm{m}$ all over the detector.

As in the Muon Spectrometer there are large temperature gradients (in the order of several ${ }^{\circ} \mathrm{C}$ ) and different chambers are located in very different conditions of magnetic field (from 0 to $0.6 \mathrm{~T}$ ), the $r(t)$ relations of different chambers can significantly differ. An example of the measured differences for several barrel chambers is shown in Fig. 9, showing differences as large as $200 \mu \mathrm{m}$.

\section{The Tube Spatial Resolution.}

Final performance of the MDT chambers and quality of the calibration constants can be evaluated from the measurement of the tube spatial resolution as a function of the distance from the wire (the drift radius). In Fig. 10 MDT drift tube
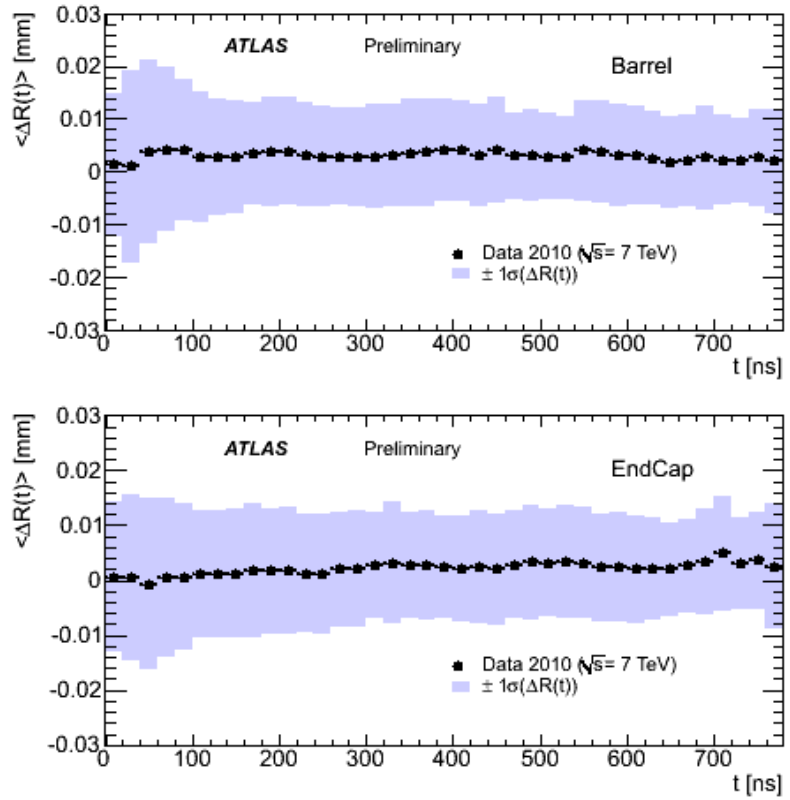

Fig. 8. Difference between the $r(t)$ relations measured in different high statistics runs taken few days apart in October 2010 [9].

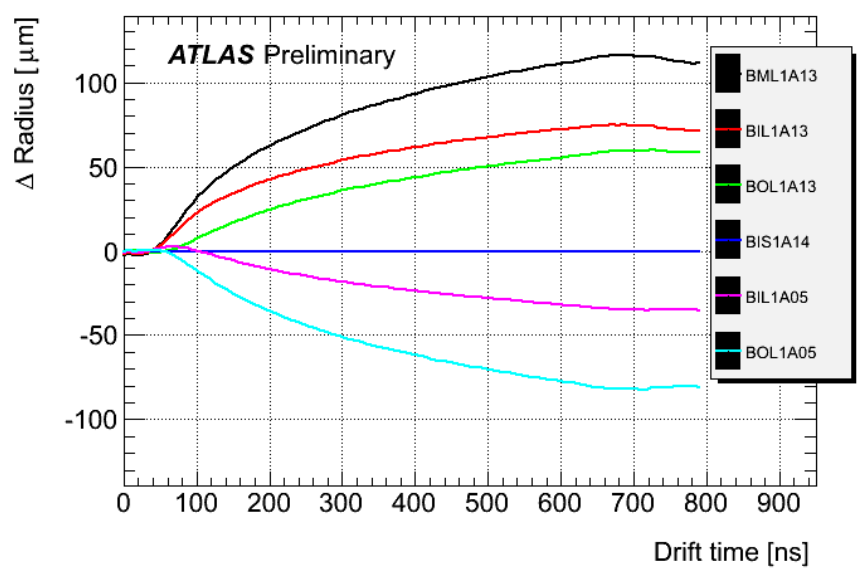

Fig. 9. Differences between $r(t)$ relations (in the same run) for several barrel chambers. [9]

spatial resolution as a function of the radius is reported. It was measured separately for Inner (red squares), Middle (black triangles), Outer (green triangles) chambers of the Muon Spectrometer barrel side A (similar results are obtained for the other regions of the MS). Hits on segments associated to tracks with transverse momenta larger than $15 \mathrm{GeV}$ were used in the analysis for a run in July 2011. The spatial resolution of the tube was measured with the following procedure. Track segments with at least 6 hits are selected. The segments are refitted leaving out one of the hit tubes. The spatial resolution is measured from the width of the hit residual distribution for hits removed from the segment fit. The segment fit error extrapolated to the tube under investigation is subtracted in quadrature from the residual width. A systematic uncertainty of $10 \mu \mathrm{m}$ is estimated for each measured point. An average of $126 \mu \mathrm{m}$ is obtained. 


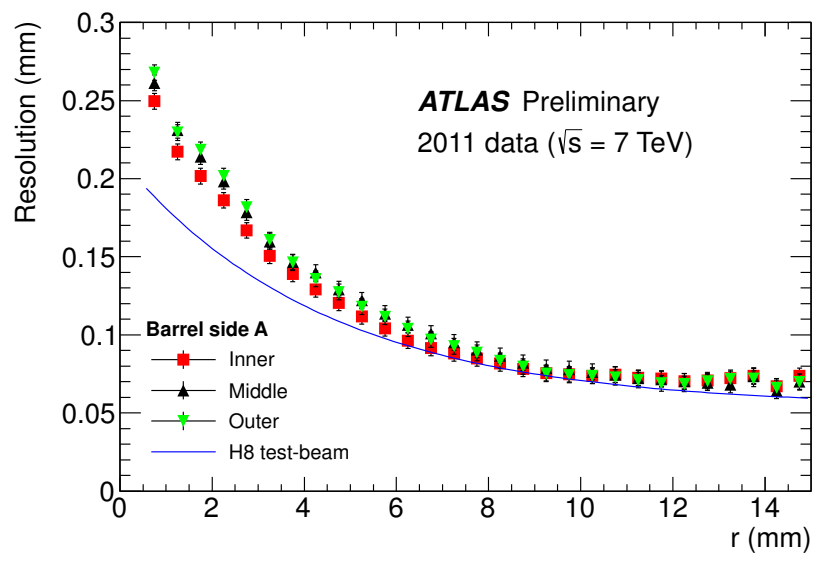

Fig. 10. MDT hit spatial resolution as a function of tube radius for barrel (side A) chambers [9]

The solid line in the figure represents the fit to the resolution measured with a high momentum muon beam, on a small portion of a single chamber (using a $10 \times 10 \mathrm{~cm}^{2}$ trigger) averaging over few tubes with optimal single tube calibrations [7]. This resolution curve resulted to be in good agreement with GARFIELD simulation. An average resolution of about 100 $\mu \mathrm{m}$ was obtained.

\section{Conclusions.}

Calibrations for the precision chambers of the ATLAS Muon spectrometer are performed daily with a high statistics muon calibration stream extracted from the second level of trigger.

Continuous monitoring of the quality of the data is performed down to the level of individual drift tubes.

The calibrations of timing offset $t_{0}$ 's and $r(t)$ functions provide an average $126 \mu \mathrm{m}$ single tube resolution (design specs $100 \mu \mathrm{m})$.

Muon momentum resolution is dominated at large momenta by alignment and intrinsic spatial resolution. Fig. 11 shows a very nice example of an analysis performed with very high transverse momentum muons for searches of $Z^{\prime}$ narrow resonance (predicted by several extensions of the Standard Model). The figure shows the dimuon invariant mass distribution compared to the stacked sum of all expected backgrounds, with three example $Z^{\prime}$ signals overlaid [8].

The good performance achieved for the ATLAS Muon Spectrometer and in particular in the calibration of the precision tracking chambers, make it possible to explore physics to discoveries at energies above the $\mathrm{TeV}$ region.

\section{REFERENCES}

[1] The ATLAS Technical Proposal, CERN-LHCC/94-43, 15.12.1994.

[2] The ATLAS Collaboration, G. Aad et al., The ATLAS Experiment at the CERN Large Hadron Collider, JINST 3, S08003, 2008.

[3] The ATLAS Collaboration, G. Aad et al., Expected Performance of the ATLAS Experiment: Detector, Trigger and Physics, CERN-OPEN 2008020, December 2008, ISBN 978-92-9083-321-5.

[4] M. Livan, Nucl. Instr. and Meth. in Phys. Res. A384 (1996) 214; F. Bauer et al., Nucl. Instr. and Meth. in Phys. Res. A461 (2001)17.

[5] P.Bagnaia, et al., "Calibration model for the MDT chambers of the ATLAS Muon Spectrometer, ATL-MUON-PUB-2008-004;

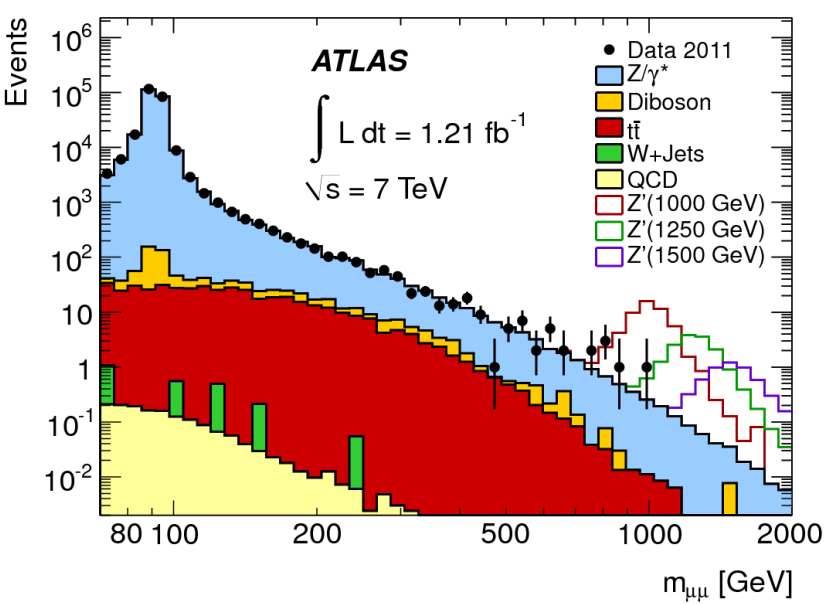

Fig. 11. Dimuon invariant mass

[6] E.Pasqualucci, et al. "Muon detector calibration in the ATLAS experiment: data extraction and distribution", Proceedings of Computing In High Energy and Nuclear Physics CHEP 2006.

[7] C. Adorisio et al. System Test of the ATLAS Muon Spectrometer in the H8 Beam at the CERN SPS, Nucl. Instrum. Meth. A 593, 232-254, 2008.

[8] ATLAS Collaboration, Search for dilepton resonances in pp collisions at sqrt(s) $=7 \mathrm{TeV}$ with the ATLAS detector, arXiv:1108.1582v2 [hep-ex], accepted by PRL

[9] http://atlas.web.cern.ch/Atlas/GROUPS/MUON/PLOTS/ATLAS-PLOTMUON-2011-006/ 